\title{
SBEVNet: End-to-End Deep Stereo Layout Estimation
}

\author{
Divam Gupta \\ Carnegie Mellon University \\ divamecmu.edu \\ Trenton Tabor \\ National Robotics Engineering Center \\ ttaboranrec.ri.cmu.edu
}

\author{
Wei $\mathrm{Pu}$ \\ National Robotics Engineering Center \\ wpu@nrec.ri.cmu.edu
}

Jeff Schneider

Carnegie Mellon University

schneidedcs. cmu.edu

\begin{abstract}
Accurate layout estimation is crucial for planning and navigation in robotics applications, such as self-driving. In this paper, we introduce the Stereo Bird's Eye View Network (SBEVNet) 1 a novel supervised end-to-end framework for estimation of bird's eye view layout from a pair of stereo images. Although our network reuses some of the building blocks from the state-of-the-art deep learning networks for disparity estimation, we show that explicit depth estimation is neither sufficient nor necessary. Instead, the learning of a good internal bird's eye view feature representation is effective for layout estimation. Specifically, we first generate a disparity feature volume using the features of the stereo images and then project it to the bird's eye view coordinates. This gives us coarse-grained information about the scene structure. We also apply inverse perspective mapping (IPM) to map the input images and their features to the bird's eye view. This gives us fine-grained texture information. Concatenating IPM features with the projected feature volume creates a rich bird's eye view representation which is useful for spatial reasoning. We use this representation to estimate the BEV semantic map. Additionally, we show that using the IPM features as a supervisory signal for stereo features can give an improvement in performance. We demonstrate our approach on two datasets: the KITTI [5] dataset and a synthetically generated dataset from the CARLA [4] simulator. For both of these datasets, we establish state-of-the-art performance compared to baseline techniques.
\end{abstract}

\section{Introduction}

Layout estimation is an extremely important task for navigation and planning in numerous robotics applications such as autonomous driving cars. The bird's eye view

${ }^{1}$ The code is available at/https://github.com/divamgupta/ sbevnet-stereo-layout-estimation

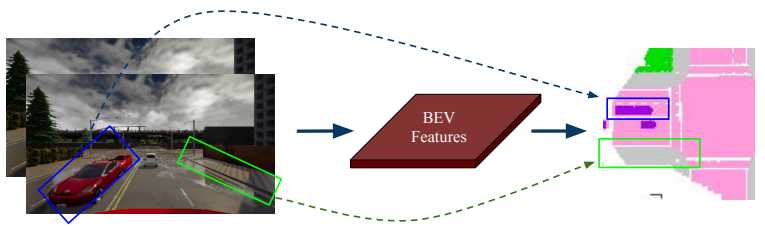

Stereo Image Pair

BEV Representation BEV Map Prediction

Figure 1. SBEVNet estimates a semantic layout in the bird's eye view space from a stereo image pair. The semantic map contains regions of the scene such as vegetation, cars, road, sidewalk, etc. $S B E V N e t$ first creates a BEV representation by leveraging the rules camera geometry. This BEV representation is then used to estimate the BEV semantic map via a U-Net model.

(BEV) layout is a $2 \mathrm{D}$ semantic occupancy map containing per pixel class information, e.g. road, sidewalk, cars, vegetation, etc. The BEV semantic map is important for planning the path of the robot in order to prevent it from hitting objects and going to impassable locations.

In order to generate a BEV layout, we need 3D information about the scene. Sensors such as LiDAR (Light Detection And Ranging) can provide accurate point clouds. The biggest limitations of LiDAR are high cost, sparse resolution, and low scan-rates. Also, as an active sensor LiDAR is more power hungry, more susceptible to interference from other radiation sources, and can affect the scene. Cameras on the other hand, are much cheaper, passive, and capture much more information at a higher frame-rate. However, it is both hard and computationally expensive to get accurate depth and point clouds from cameras.

The classic approach for stereo layout estimation contains two steps. The first step is to generate a BEV feature map by an orthographic projection of the point cloud generated using stereo images. The second step is bird's eye view semantic segmentation using the projected point cloud from the first step. This approach is limited by the estimated point 
cloud accuracy because the error in it will propagate to the layout estimation step. In this paper, we show that explicit depth estimation is actually neither sufficient nor necessary for good layout estimation. Estimating accurate depth is not sufficient because many areas in the 3D space can be occluded partially, e.g. behind a tree trunk. However, these areas can be estimated by combining spatial reasoning and geometric knowledge in bird's eye view representation. Explicitly estimating accurate depth is also not necessary because layout estimation can be done without estimating the point cloud. Point cloud coordinate accuracy is limited by the $3 \mathrm{D}$ to $2 \mathrm{D} \mathrm{BEV}$ projection and rasterization. For these reasons, having an effective bird's eye view representation is very important.

SBEVNet is built upon recent deep stereo matching paradigm. These deep learning based methods have shown tremendous success in stereo disparity/depth estimation. Most of these models [11, 9, 21, 19, 6, 26, 2, 8] generate a 3dimensional disparity feature volume by concatenating the left and right images shifted at different disparities, which is used to make a cost volume containing stereo matching costs for each disparity value. Given a location in the image and the disparity, we can get the position of the corresponding $3 \mathrm{D}$ point in the world space. Hence, every point in the feature volume and cost volume corresponds to a 3D location in the world space. The innovation in our approach comes from the observation: it is possible to directly use the feature volume for layout estimation, rather than a two step process, which uses the point cloud generated by the network. We propose SBEVNet, an end-to-end neural architecture that takes a pair of stereo images and outputs the bird's eye view scene layout. We first project the disparity feature volume to the BEV view, creating a $2 \mathrm{D}$ representation from the 3D volume. We then warp it by mapping different disparities and the image coordinates to the bird's eye view space. In order to overcome the loss of fine grained information imposed by our choice of the stereo BEV feature map, we concatenate a projection of the original images and deep features to this feature map. We generate these projected features by applying inverse perspective mapping (IPM) [13] to the input image and its features, choosing the ground as the target plane. We feed this representation to a U-Net in order to estimate the BEV semantic map of the scene.

In order to perform inverse perspective mapping, we require information about the ground in the 3D world space. Hence we also consider the scenario where we perform IPM during the training time and not the inference time. Here, during the training time, we use cross modal distillation to transfer knowledge from IPM features to the stereo features.

SBEVNet is the first approach to use an end-to-end neural architecture for stereo layout estimation. We show that $S B E V N e t$ achieves better performance than existing ap- proaches. SBEVNet outperforms all the baseline algorithms on KITTI [5] dataset and a synthetically generated dataset extracted from the CARLA simulator [4]. In summary, our contributions are the following:

1. We propose SBEVNet, an end-to-end neural architecture for layout estimation from a stereo pair of images.

2. We learn a novel representation for BEV layout estimation by fusing projected stereo feature volume and fine grained inverse perspective mapping features.

3. We evaluate SBEVNet and demonstrate state-of-theart performance over other methods by a large margin on two datasets - KITTI dataset and our synthetically generated dataset using the CARLA simulator.

\section{Related Work}

To the best of our knowledge, there is no published work for estimating layout given a pair of stereo images. However, there are several works tackling layout estimation using a single image or doing object detection using stereo images. In this section, we review the most closely related approaches.

Monocular Layout Estimation MonoLayout [14] uses an encoder-decoder model to estimate the bird's eye view layout using a monocular input image. They also leverage adversarial training to produce sharper estimates. MonoOccupancy [12] uses a variational encoder-decoder network to estimate the layout. Both MonoLayout and MonoOccupancy do not use any camera geometry priors to perform the task. Schulter et al. [16] uses depth estimation to project the image semantics to bird's eye view. They also use Open Street Maps data to refine the BEV images via adversarial learning. Wang et al. [22] uses Schulter et al. [16] to estimate the parameters of the road such as lanes, sidewalks, etc. Monocular methods learn strong prior, which does not generalize well when there is a significant domain shift. Stereo methods learn weak-prior plus geometric relationship, which can generalize better.

Deep Stereo Matching Several methods like [11, 9, 21, 19, 6, 26, 2, 8, extract the features of the stereo images and generate a 3D disparity feature volume for disparity/depth estimation. They use a 3D CNN on the feature volume to get cost volume to perform stereo matching. PSMNet [2] uses a spatial pyramid pooling module and a stacked hourglass network to further improve the performance. Highres-stereo [24] uses a hierarchical model, creating cost volumes at multiple resolutions, performing the matching incrementally from over a coarse to fine hierarchy.

Bird's Eye View Object Detection Several approaches [23, 17] use LiDAR to perform 3D object detection. Pseudo-lidar [20] and pseudo-LiDAR++ [25] use stereo input to first generate a $3 \mathrm{D}$ point cloud and then use a $3 \mathrm{D}$ ob- 
ject detection network [10, 23, 17] on top. BirdGAN [18] maps the input image to bird's eye view using adversarial learning. The closest work to our approach is DSGN [3] which constructs a depth feature volume and map it to the 3D space which is then projected to bird's eye view to perform object detection. The task of object detection is of sparse prediction, whereas layout estimation is of dense fine granularity prediction. Hence we introduced IPM to fuse low level detail with the stereo information to improve the performance of layout estimation.

\section{Our Method}

This section describes the detailed architecture of our proposed framework. SBEVNet is built upon recent deep stereo matching paradigms and follows the rules of multiview camera geometry. An overview of the SBEVNet is summarized in Figure 2 .

\subsection{Problem Formulation}

In this paper, we address the problem of layout estimation from a pair of stereo images. Formally, given a reference camera image $I_{R}$ and a target camera image $I_{T}$ both of size $H \times W \times 3$, the camera intrinsics $K$, and the baseline length $T_{b}$, we aim to estimate the bird's eye view layout of the scene. In particular, we estimate the BEV semantic map of size $N_{x} \times N_{y} \times N_{C}$ within the rectangular range of interest area $\left(x_{\min }, x_{\max }, y_{\min }, y_{\max }\right)$ in front of the camera. Here $H$ is image height, $W$ is image width, and 3 indicates RGB channels. $N_{x}$ and $N_{y}$ are the number of horizontal cells and vertical cells respectively in bird's eye view. $N_{C}$ is the number of semantic classes. This BEV semantic map contains the probability distribution among all semantic classes at each cell of the layout. We assume that the input images are rectified.

\subsection{Feature extraction}

The first step for $S B E V N e t$ is to extract features $F_{R}$ and $F_{T}$ of size $H^{\prime} \times W^{\prime} \times C$ for the reference image and the target image respectively. This is done by passing $I_{R}$ and $I_{T}$ through a convolutional encoder with shared weights. This produces multi-channel down-sized feature representations which are next used for building disparity feature volumes.

\subsection{Disparity Feature Volume Generation}

Similar to [11, 9, 21, 19, 6, 26, 2, 8] we form a disparity feature volume $V$ by concatenating the features $F_{R}$ and $F_{T}^{d}$, where $F_{T}^{d}$ is $F_{T}$ shifted horizontally by a disparity of $d$ pixel, resulting in a 3D volume of size $H^{\prime} \times W^{\prime} \times D \times 2 C$. We then pass the feature volume through a series of $3 \mathrm{D}$ convolution layers with skip connections to learn higher level features. This feature volume at each $d \in\{0,1, \cdots, D-1\}$ contains a representation of the $3 \mathrm{D}$ world at the depth corresponding to the disparity $d$. Rather than using this feature volume to do disparity estimation, we project and warp it to form a bird's eye view representation in the next step.

\subsection{Bird's Eye View Representation}

The bird's eye view representation is composed of two parts -1) The stereo BEV representation which is derived from the disparity feature volume, 2) The IPM BEV representation which is the result of applying inverse perspective mapping on the reference image and the features of the reference image. These two parts are concatenated to form the final bird's eye view representation.

\subsubsection{Stereo BEV Representation}

The disparity feature volume generated is widely used to estimate depth/disparity in the stereo image pairs. But this feature volume contains a lot of information about the 3D scene which can be used for other tasks as well. Each point in the disparity feature volume corresponds to a point in the 3D world space. We first need to map the 3D feature volume to a 2D feature map containing information of the bird's eye view. If we do max/average pooling along height dimension, a certain degree of the height information is lost quickly before being extracted for our task, which is not desirable. Considering height information a good prior for layout estimation but we don't need to recover it explicitly, we concatenate the feature volume along the height, creating a $2 \mathrm{D}$ image of size $W^{\prime} \times D \times 2 C H^{\prime}$. We then use $2 \mathrm{D}$ convolutions to generate the reduced feature volume of size $W^{\prime \prime} \times D^{\prime \prime} \times C^{\prime}$. This reduced feature volume does not spatially match with the bird's eye view layout. Hence, we warp the reduced feature volume, transforming it to a feature map of size $N_{x} \times N_{y} \times C^{\prime}$ in the bird's eye view space. Given the disparity $d$, position in the image along width $u$, camera parameters $f, c_{x}, c_{y}$, and stereo baseline length $T_{x}$, we can find the coordinates in the bird's eye space $x^{\prime}$ and $y^{\prime}$ as follows:

$$
\begin{gathered}
x^{\prime}=\frac{\left(u-c_{x}\right) \cdot T_{x}}{d} \\
y^{\prime}=\frac{f \cdot T_{x}}{d}
\end{gathered}
$$

The 2D origin of the bird's eye view is co-located with the reference camera. An example visualization of the layout in the disparity volume space is shown in Figure 3. After mapping the coordinates to the BEV space, we map them to a grid of size $N_{x} \times N_{y}$ giving us the stereo BEV representation $R_{\text {stereo. }}$.

\subsubsection{IPM BEV Representation}

The stereo BEV representation contains structural information for the bird's eye view space. Due to the refinement and 


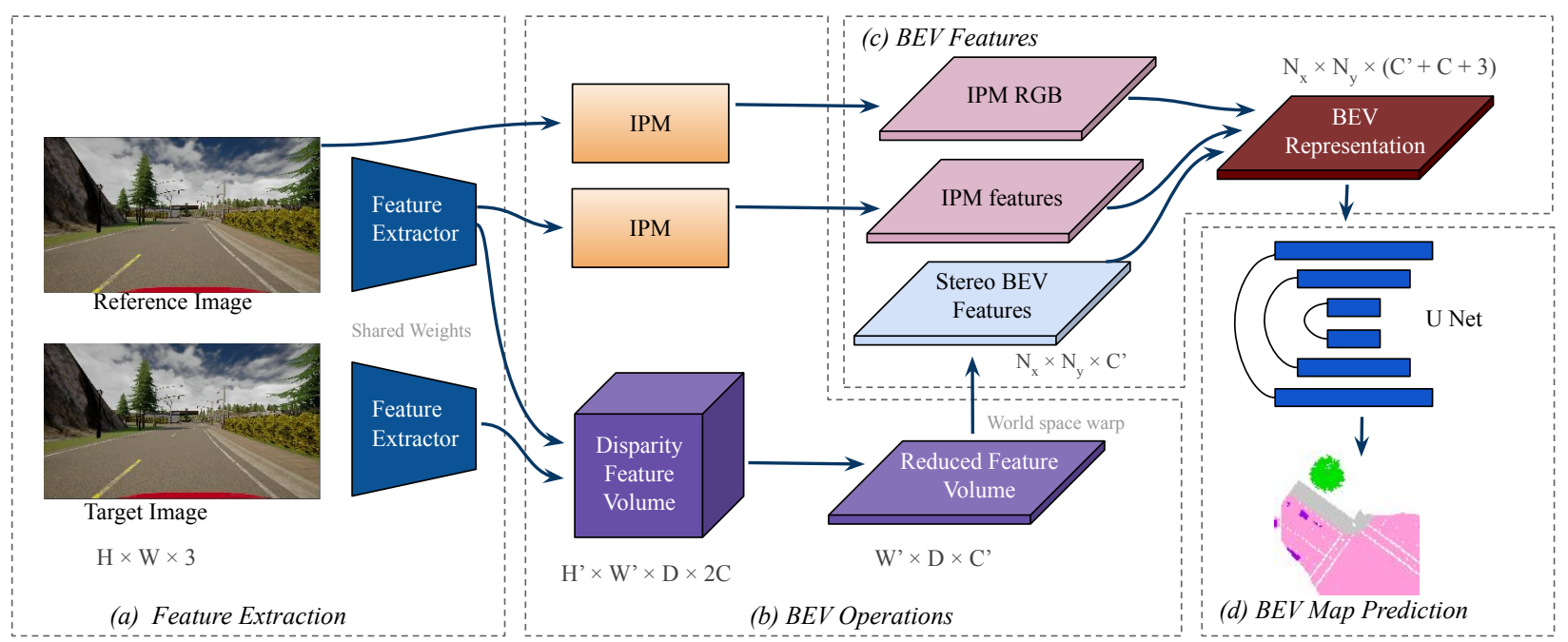

Figure 2. SBEVNet overview. We first extract the image features given the target and reference image. Using the pair of features, we create a disparity feature volume. We then reduce the disparity feature volume along with the height and warp it in the bird's eye view layout space. On a parallel branch of the network, we apply inverse perspective mapping (IPM) on the reference image and its features. We concatenate the IPM RGB, IPM feature, and the stereo BEV features. The BEV representation is then used to estimate the semantic map through a U-Net. Visibility mask is used to apply the supervised loss only at the locations in the BEV which are in the view of the front camera.

reduction of the feature volume, the fine grained details are excluded by design. To circumvent that, we need to fuse the low level features to the stereo BEV features, while maintaining geometric consistency.

In order to fuse the image features to the stereo BEV features at the correct locations, we need to warp the image features to the BEV space. We apply inverse perspective mapping on the reference image and the features of the reference image to do that.

A point in the image $I_{R}$ can correspond to multiple points in the 3D world space due to perspective projection, but there is a single point which also intersects with the ground plane. Let $z=a x+b y+c$ be the equation of the ground plane in the world space. Given the input image coordinates $(u, v)$ and camera parameters $f, c_{x}, c_{y}$, we can find the coordinates in the bird's eye space $x^{\prime}$ and $y^{\prime}$ as follows:

$$
\begin{aligned}
x^{\prime} & =\frac{c u-c c_{x}}{a c_{x}-a u-b f-c_{y}+v} \\
y^{\prime} & =\frac{c f}{a c_{x}-a u-b f-c_{y}+v}
\end{aligned}
$$

This can be easily derived by combining the camera projection equation with the equation of the ground plane. For many applications, the ground is either planar or can be approximated by a plane. This is also equivalent to computing a homography $H$ between the ground plane and the image plane of the layout and then applying the transformation.
We can have the parameters of the plane $a, b$, and $c$ predetermined if the placement of the camera with respect to the ground is known, which is the case for many robotics applications. We can also determine $a, b$ and $c$ by using stereo depth and a semantic segmentation network for the $\mathrm{road} /$ ground class.

Examples of IPM on the input images is shown in Figure 3 We apply the inverse perspective transform on both the input image and the features of the input image to transform them to the bird's eye view space:

$$
\begin{aligned}
& R_{\text {IPM_feat }}=\operatorname{IPM}\left(F_{R}\right) \\
& R_{\text {IPM_img }}=\operatorname{IPM}\left(I_{R}\right)
\end{aligned}
$$

They are then concatenated with the stereo BEV representation to form the combined BEV representation:

$$
R_{\mathrm{BEV}}=\left[R_{\mathrm{IPM} \_ \text {feat }} ; R_{\mathrm{IPM} \_ \text {img }} ; R_{\text {stereo }}\right]
$$

\subsubsection{IPM for cross modal distillation}

There can be use-cases where we cannot do inverse perspective mapping during inference time, due to the unavailability of the ground information. Hence, we consider the case where IPM is only available during the training time. We can think of the IPM features and the stereo features as different modalities and apply cross modal distillation [7] across them, and transfer knowledge from IPM features 
to the stereo features. Hence, we use the IPM BEV representation as a supervisory signal for the stereo BEV features. This forces the stereo branch of the model to implicitly learn the fine grained information learned by the IPM features. Rather than concatenating the IPM BEV features with the stereo features, we minimize the distance between them. We call this variant of SBEVNet as SBEVNet-CMD (SBEVNet cross modal distillation). During the training time, the IPM BEV features and the stereo features are used to generate the BEV semantic maps.

$$
\begin{aligned}
& C^{\mathrm{IPM}}=\mathrm{U}-\mathrm{Net}\left(R_{\text {IPM_feat }}\right) \\
& C^{\text {stereo }}=\mathrm{U}-\operatorname{Net}\left(R_{\text {stereo }}\right)
\end{aligned}
$$

This ensures both IPM BEV features and stereo BEV features learn meaningful information. We jointly minimise the $L_{1}$ distance between first $K$ channels of the features.

$$
L_{\mathrm{KT}}=\left\|R_{\text {IPM_feat }}[: K]-R_{\text {stereo }}[: K]\right\|_{L_{1}}
$$

By this, we ensure that the stereo model can learn information that is not in the IPM features. In our experiments, we found this to yield better results compared to the approach of minimizing the $L_{1}$ distance between all the channels of the features. During test time, we only use the stereo features to get the BEV layout. Our experiments show that the stereo model with cross modal distillation performs better than the stereo model without cross modal distillation.

\subsubsection{Layout Generation}

We can generate the semantic map by inputting the BEV features to a semantic segmentation network. We pass the concatenated stereo BEV feature map and IPM BEV feature map to a U-Net [15] network to generate the semantic map C.

$$
C=\mathrm{U}-\mathrm{Net}\left(R_{\mathrm{BEV}}\right)
$$

Some areas in the layout may not be in the view of the front camera, e.g. things behind a wall. That is why it is not a good idea to penalize the model for the wrong prediction for those areas. Hence, we use a visibility mask to mask the pixel-wise loss, applying it only on the pixels which are in the field of view. This mask is generated during the ground truth generation process by using ray-tracing on the point cloud to determine which are in the field of view. For a visibility mask $V, V_{i}$ is 1 if the pixel $i$ is in the view of the input image, and 0 otherwise. For the loss, we use a pixelwise categorical cross entropy loss as follows:

$$
L_{r}=\sum_{i \in P} V_{i} \cdot \operatorname{CE}\left(C_{i}, C_{i}^{h}\right)
$$

where $C_{i}^{h}$ is ground truth. The total loss for SBEVNet-CMD is the sum of supervision loss from the two feature maps and the $L_{1}$ distance minimization.

$$
L_{c}=\sum_{i \in P} V_{i} \cdot \mathrm{CE}\left(C_{i}^{\mathrm{IPM}}, C_{i}^{h}\right)+\sum_{i \in P} V_{i} \cdot \mathrm{CE}\left(C_{i}^{\text {stereo }}, C_{i}^{h}\right)+L_{\mathrm{KT}}
$$

\section{Experiments}

\subsection{Datasets}

CARLA dataset: We use the CARLA [4] simulator to generate a synthetic dataset, containing 4,000 and 925 training and testing data points respectively. The bounds of the layout with respect to the camera are -19 to 19 meters in $x$ direction and 1 to 39 meters in the $y$ direction.

KITTI dataset: We also evaluate SBEVNet on the odometery subset of the KITTI [5] dataset. We use the SemanticKITTI [1] dataset for labeled ground truth.

\subsection{Evaluation Metrics}

As not all the regions of the ground truth layout are visible from the camera, we only consider pixels of the layout which are in the field of view. For evaluating the semantic map, we use macro averaged intersection over union (IoU) scores for the layout pixels which are in the visibility mask. We report the IoU scores for each semantic class separately.

\subsection{Compared Methods}

There are no previously reported quantitative results for the task of stereo layout estimation in our setting. Thus, we evaluate appropriate baselines which are prior works extended to our task.

1. Pseudo-LiDAR [20] + segmentation: Uses Pseudolidar with PSMNet to generate a 3D point cloud from the input stereo images which is used to project the semantic segmentation of the front view to the bird's eye view. The PSMNet is trained separately on the respective datasets for better performance.

2. Pseudo-LidAR [20] + BEV U-Net: The RGB 3D point projected in the BEV aligned with the ground truth layout is used to train a U-Net segmentation network.

3. IPM + BEV U-Net: Inverse perspective mapping is applied to the input image to project it to the BEV space which is used to train a U-Net segmentation network.

4. MonoLayout [14]: This baseline uses MonoLayout to generate BEV semantic map from a single image. Rather than using OpenStreetMap data for adversarial training, we used random samples from the training set itself. 

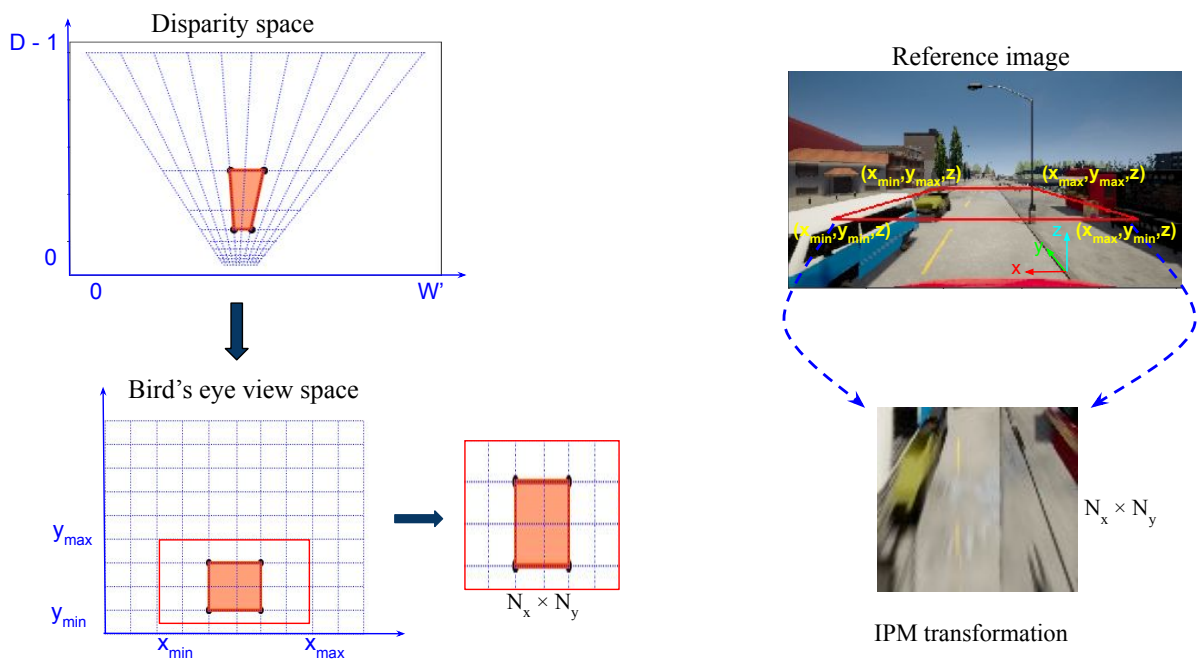

Figure 3. Illustration of mapping the disparity space to bird's eye view space and inverse perspective mapping. (a) The operation maps different disparities and $x$ to the BEV space in order to match the ground truth. We also show an example layout warped to the disparity space. (b) The inverse perspective mapping operation maps pixels of the reference image to the BEV space in order to match the ground truth. The same mapping can be applied to the image features as well.

\begin{tabular}{c|c|ccccc}
\hline Method & mIoU & Road & Vegetation & Cars & Sidewalk & Building \\
\hline \hline Pseudo-LiDAR + segmentation & 25.63 & 37.64 & 16.40 & 35.15 & 25.44 & 13.50 \\
Pseudo-LiDAR + BEV U-Net & 36.61 & 63.55 & 31.87 & 45.64 & 29.97 & 12.01 \\
IPM + BEV U-Net & 32.30 & 66.36 & 15.24 & 41.37 & 32.77 & 5.78 \\
MonoLayout & 22.16 & 52.88 & 9.00 & 16.36 & 23.17 & 9.41 \\
MonoLayout + depth & 21.85 & 52.94 & 9.95 & 14.07 & 23.02 & 9.31 \\
MonoOccupancy + depth & 29.49 & 67.96 & 16.56 & 7.66 & 36.35 & 18.91 \\
\hline SBEVNet only stereo & 36.10 & 64.74 & 31.85 & 39.76 & 30.01 & 14.14 \\
SBEVNet stereo + RGB IPM & 39.77 & 65.01 & $\mathbf{3 3 . 2 0}$ & 47.88 & 33.24 & 19.53 \\
SBEVNet stereo + features IPM & 42.29 & 71.29 & 29.79 & 51.97 & 38.46 & 19.95 \\
SBEVNet-CMD & 40.10 & 69.07 & 32.71 & 45.45 & 35.16 & 18.08 \\
SBEVNet & $\mathbf{4 4 . 3 6}$ & $\mathbf{7 2 . 8 2}$ & 32.07 & $\mathbf{5 5 . 3 2}$ & $\mathbf{4 0 . 6 9}$ & $\mathbf{2 0 . 7 8}$ \\
\hline SBEVNet Ensemble & $\mathbf{4 7 . 9 2}$ & $\mathbf{7 5 . 3 6}$ & $\mathbf{3 5 . 3 3}$ & $\mathbf{6 0 . 1 7}$ & $\mathbf{4 4 . 2 5}$ & $\mathbf{2 4 . 4 7}$ \\
\hline TBE
\end{tabular}

Table 1. Quantitative results of semantic layout estimation on the CARLA dataset.

5. MonoLayout [14] + depth: The input RGB image concatenated with the depth is used as an input to the MonoLayout Model.

6. MonoOccupancy [12] + depth: The input RGB image concatenated with the depth is used as an input to the MonoOccupancy Model.

We also evaluate some variations of our model to perform ablation studies. In SBEVNet only stereo we exclude the IPM features and only use features derived from the feature volume. To gauge the importance of IPM on RGB images and features, we also try applying IPM only on RGB images (SBEVNet stereo + RGB IPM) and IPM only on the features of the input image (SBEVNet stereo + features IPM). We also evaluate the cross modal distillation model
SBEVNet-CMD. Finally, we evaluate our complete model (SBEVNet) where we use stereo features and IPM on both RGB image and its features. We also evaluate SBEVNet Ensemble where we take an ensemble of SBEVNet with the same architecture but different initialization seeds.

\subsection{Implementation Details}

We implemented SBEVNet using Pytorch. We use Adam optimizer with the initial learning rate of 0.001 and betas $(0.9,0.999)$ for training. We use a batch-size of 3 on a Titan $\mathrm{X}$ Pascal GPU. We use the same base network which is used in the basic model of PSMNet. The input image size for the CARLA dataset is $512 \times 288$ and the input image size for the KITTI dataset is $640 \times 256$. We report the average scores according to 8 runs to account for the stochasticity due to ran- 


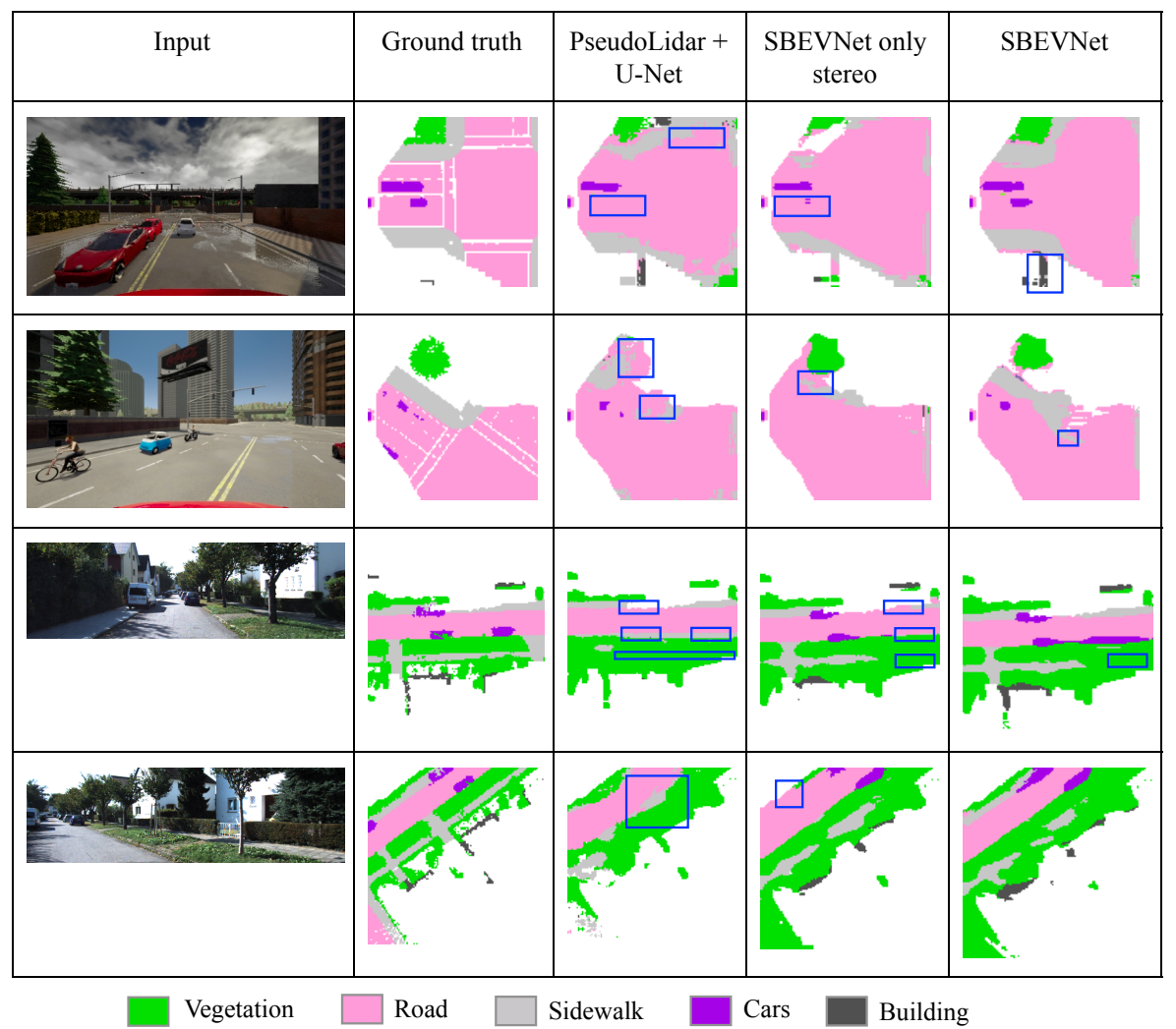

Figure 4. Qualitative results on the test set of the CARLA and the KITTI dataset. The major mistakes in the predictions are annotated by a blue rectangle.

\begin{tabular}{c|c|ccccc}
\hline Method & mIoU & Road & Sidewalk & Cars & Building & Vegetation \\
\hline \hline Pseudo-LiDAR + segmentation & 18.69 & 35.51 & 14.56 & 13.50 & 12.64 & 17.26 \\
Pseudo-LiDAR + BEV U-Net & 28.97 & 61.83 & 21.36 & 5.55 & 12.74 & 43.38 \\
IPM + BEV U-Net & 34.93 & 68.40 & 26.65 & 28.49 & 4.53 & 46.57 \\
MonoLayout & 25.19 & 64.36 & 20.53 & 2.43 & 2.59 & 36.05 \\
MonoLayout + depth & 21.48 & 55.80 & 16.19 & 1.91 & 3.03 & 30.46 \\
MonoOccupancy + depth & 29.16 & 70.52 & 22.17 & 7.11 & 5.25 & 40.77 \\
\hline SBEVNet only stereo & 50.01 & 78.41 & 40.16 & 41.96 & 30.45 & 59.05 \\
SBEVNet stereo + RGB IPM & 49.56 & 78.37 & 39.83 & 42.47 & 28.34 & 58.80 \\
SBEVNet stereo + features IPM & 50.60 & 80.16 & 41.08 & $\mathbf{4 3 . 6 4}$ & 29.19 & 58.92 \\
SBEVNet -CMD & 50.73 & $\mathbf{8 0 . 5 9}$ & 41.67 & 43.16 & 29.13 & 59.37 \\
SBEVNet & $\mathbf{5 1 . 3 6}$ & 80.23 & $\mathbf{4 1 . 8 6}$ & 42.81 & $\mathbf{3 1 . 3 5}$ & $\mathbf{5 9 . 4 3}$ \\
\hline SBEVNet Ensemble & $\mathbf{5 3 . 8 5}$ & $\mathbf{8 2 . 2 2}$ & $\mathbf{4 5 . 7 0}$ & $\mathbf{4 4 . 9 7}$ & $\mathbf{3 4 . 5 4}$ & $\mathbf{6 1 . 8 3}$ \\
\hline Table 2. Quantitative results of semantic layout estimation on the KITTI dataset
\end{tabular}

dom initialization and other non-deterministic operations in the network.

\subsection{Experimental results}

We report the IoU scores of all the methods on the CARLA and KITTI [5] dataset in Table 1 and Table 2 respectively. As we can see from the tables, SBEVNet achieves superior performance on both the datasets. We also observe the increase in performance if we use both stereo information and inverse perspective mapping. IPM yields a greater increase in performance in the CARLA [4] dataset because the ground is perfectly flat. If we use only RGB IPM along with stereo, the results are slightly worse on the KITTI dataset because the ground is not perfectly planar. We see that degradation does not persist if we also use IPM on the image features. For the KITTI dataset, we 
see a sharp improvement over pseudo-LiDAR approaches because of inaccurate depth estimation. On the other hand, our model does not depend on explicit depth data/model. The results of MonoLayout [14] and MonoOccupancy [12] are inferior due to lack of any camera geometry priors in the network. We also show the qualitative results on the test set of CARLA [4] and KITTI [5] dataset in Figure 4] We see that in certain regions $S B E V N e t$ gives outputs closer to the ground truth. For example, Psuedo-lidar fails to segment cars in the KITTI dataset. We also observe a drop in quality in the estimated layout as we move further from the camera.

\subsubsection{Ablation Study}

IPM on RGB image For the CARLA dataset, we observe an increase of 3.67 in the mIoU score, on concatenating IPM RGB with the stereo features. We observe an increase in IoU scores for all the classes, with the biggest increase of 8.12 in the cars class. For the KITTI dataset, there is a small decrease of 0.45 in the mIoU score. This is because, the ground is not perfectly planar, hence the IPM RGB images do not exactly align with the ground truth layout.

IPM on image features If we apply IPM on the features of the input image and concatenate it with the features from the stereo branch, we see an improvement in both the datasets. The improvements in mIoU scores are 6.19 and 0.59 for the CARLA and KITTI dataset. The improvement is higher compared to the RGB IPM because image features contain higher level information which is transformed to the BEV space.

IPM on both RGB image and image features We see the greatest improvement if we apply IPM on both the RBG image and the features of the RGB image. The improvements in mIoU scores are 8.26 and 1.35 for the CARLA and KITTI dataset respectively. This is because the model is able to exploit the different information present in $R_{\text {IPM_feat }}$ and $R_{\text {stereo }}$.

Cross modal distillation The performance of SBEVNetCDM is in between of stereo only SBEVNet and full SBEVNet. We see an improvement of 4.00 and 0.72 in the mIoU scores on the CARLA and KITTI dataset, if we train the stereo model using cross modal distillation via IPM features. During inference, the architecture of SBEVNet-CMD is the same as the stereo only SBEVNet. This shows that CMD is able to transfer most of the IPM knowledge to the stereo branch.

Minimizing distance between first $K$ features We also evaluate the approach, where we try minimizing the L1 distance between all the channels of the IPM features and stereo features. We observe mIoU scores of 32.27 and 50.03 for the CARLA and KITTI dataset respectively. This is worse than the mIoU scores achieved by minimizing the distance between first $K$ channels. This is because, if we enforce all stereo branch channels to be the same as IPM branch channel, the stereo branch is unable to learn information that is not present in the IPM features.

\subsection{Discussions}

\subsubsection{Distance from camera}

We wish to quantify how our system performs as we move away from the camera. Hence, we see the IoU scores for the pixels in the BEV layout which are more than a given distance from the camera and for the pixels which are less than a given distance from the camera. For both the KITTI and the CARLA dataset, we observe that there is a drop in performance as the distance from the camera increases. We also observe that $S B E V N e t$ outperforms the stereo only $S B E V N e t$ at all distances from the camera.

\subsubsection{D feature volume analysis}

One claim of our approach is that our model learns 3D information without any explicit depth/disparity supervision. To validate this claim, we use the learned 3D feature volume to perform disparity estimation. We freeze all the weights and add a small 3D convolution layer to perform disparity regression on the learned feature volume. We also observe that the feature volume which is trained with cross modal distillation via IPM performs better at the task of disparity estimation. For the CARLA dataset, we find that the SBEVNet only stereo model has a 3-pixel error of $\mathbf{7 . 9 2}$ and with cross model distillation the 3-pixel error goes down to 6.84.

\subsubsection{Ensemble}

We observe some variance in the performance of the models on training with different random seeds. For SBEVNet we observe a standard deviation of 2.16 and 2.46 in the mIoU scores for the KITTI and CARLA dataset respectively. Due to the diversity in outputs of the individual models ([27]), we see an improvement in the performance, if we take an ensemble of individual models. We observe an absolute improvement of 2.49 and 3.56 in the mIoU scores for the KITTI and CARLA dataset respectively.

\section{Conclusion}

In this paper we proposed SBEVNet, an end-to-end network to estimate the bird's eye view layout using a pair of stereo images. We observe improvement in the IoU scores compared with approaches that are not end-to-end or do not use geometry. We also showed that combining inverse perspective mapping with the projected disparity feature volume gives better performance. 


\section{References}

[1] Jens Behley, Martin Garbade, Andres Milioto, Jan Quenzel, Sven Behnke, Cyrill Stachniss, and Jurgen Gall. SemanticKITTI: A dataset for semantic scene understanding of lidar sequences. In Proceedings of the IEEE International Conference on Computer Vision, pages 9297-9307, 2019.

[2] Jia-Ren Chang and Yong-Sheng Chen. Pyramid stereo matching network. In Proceedings of the IEEE Conference on Computer Vision and Pattern Recognition, pages 54105418, 2018.

[3] Yilun Chen, Shu Liu, Xiaoyong Shen, and Jiaya Jia. DSGN: Deep stereo geometry network for $3 \mathrm{~d}$ object detection. In Proceedings of the IEEE/CVF Conference on Computer Vision and Pattern Recognition, pages 12536-12545, 2020.

[4] Alexey Dosovitskiy, German Ros, Felipe Codevilla, Antonio Lopez, and Vladlen Koltun. CARLA: An open urban driving simulator. arXiv preprint arXiv:1711.03938, 2017.

[5] Andreas Geiger, Philip Lenz, Christoph Stiller, and Raquel Urtasun. Vision meets robotics: The KITTI dataset. The International Journal of Robotics Research, 32(11):12311237, 2013.

[6] Xiaoyang Guo, Kai Yang, Wukui Yang, Xiaogang Wang, and Hongsheng Li. Group-wise correlation stereo network. In Proceedings of the IEEE Conference on Computer Vision and Pattern Recognition, pages 3273-3282, 2019.

[7] Saurabh Gupta, Judy Hoffman, and Jitendra Malik. Cross modal distillation for supervision transfer. In Proceedings of the IEEE conference on computer vision and pattern recognition, pages 2827-2836, 2016.

[8] Alex Kendall, Hayk Martirosyan, Saumitro Dasgupta, Peter Henry, Ryan Kennedy, Abraham Bachrach, and Adam Bry. End-to-end learning of geometry and context for deep stereo regression. In Proceedings of the IEEE International Conference on Computer Vision, pages 66-75, 2017.

[9] Sameh Khamis, Sean Fanello, Christoph Rhemann, Adarsh Kowdle, Julien Valentin, and Shahram Izadi. StereoNet: Guided hierarchical refinement for real-time edge-aware depth prediction. In Proceedings of the European Conference on Computer Vision (ECCV), pages 573-590, 2018.

[10] Jason Ku, Melissa Mozifian, Jungwook Lee, Ali Harakeh, and Steven L Waslander. Joint 3D proposal generation and object detection from view aggregation. In 2018 IEEE/RSJ International Conference on Intelligent Robots and Systems (IROS), pages 1-8. IEEE, 2018.

[11] Zhengfa Liang, Yiliu Feng, Yulan Guo, Hengzhu Liu, Wei Chen, Linbo Qiao, Li Zhou, and Jianfeng Zhang. Learning for disparity estimation through feature constancy. In Proceedings of the IEEE Conference on Computer Vision and Pattern Recognition, pages 2811-2820, 2018.

[12] Chenyang Lu, Marinus Jacobus Gerardus van de Molengraft, and Gijs Dubbelman. Monocular semantic occupancy grid mapping with convolutional variational encoderdecoder networks. IEEE Robotics and Automation Letters, 4(2):445-452, 2019.

[13] Hanspeter A Mallot, Heinrich H Bülthoff, JJ Little, and Stefan Bohrer. Inverse perspective mapping simplifies optical flow computation and obstacle detection. Biological cybernetics, 64(3):177-185, 1991.

[14] Kaustubh Mani, Swapnil Daga, Shubhika Garg, Sai Shankar Narasimhan, Madhava Krishna, and Krishna Murthy Jatavallabhula. MonoLayout: Amodal scene layout from a single image. In The IEEE Winter Conference on Applications of Computer Vision, pages 1689-1697, 2020.

[15] Olaf Ronneberger, Philipp Fischer, and Thomas Brox. UNet: Convolutional networks for biomedical image segmentation. In International Conference on Medical image computing and computer-assisted intervention, pages 234-241. Springer, 2015.

[16] Samuel Schulter, Menghua Zhai, Nathan Jacobs, and Manmohan Chandraker. Learning to look around objects for topview representations of outdoor scenes. In Proceedings of the European Conference on Computer Vision (ECCV), pages 787-802, 2018.

[17] Shaoshuai Shi, Xiaogang Wang, and Hongsheng Li. PointR$\mathrm{CNN}$ : 3D object proposal generation and detection from point cloud. In Proceedings of the IEEE Conference on Computer Vision and Pattern Recognition, pages 770-779, 2019.

[18] Siddharth Srivastava, Frederic Jurie, and Gaurav Sharma. Learning 2D to 3D lifting for object detection in 3d for autonomous vehicles. arXiv preprint arXiv:1904.08494, 2019.

[19] Deqing Sun, Xiaodong Yang, Ming-Yu Liu, and Jan Kautz. PWC-Net: CNNs for optical flow using pyramid, warping, and cost volume. In Proceedings of the IEEE conference on computer vision and pattern recognition, pages 8934-8943, 2018.

[20] Yan Wang, Wei-Lun Chao, Divyansh Garg, Bharath Hariharan, Mark Campbell, and Kilian Q Weinberger. PseudoLiDAR from visual depth estimation: Bridging the gap in $3 \mathrm{~d}$ object detection for autonomous driving. In Proceedings of the IEEE Conference on Computer Vision and Pattern Recognition, pages 8445-8453, 2019.

[21] Yan Wang, Zihang Lai, Gao Huang, Brian H Wang, Laurens Van Der Maaten, Mark Campbell, and Kilian Q Weinberger. Anytime stereo image depth estimation on mobile devices. In 2019 International Conference on Robotics and Automation (ICRA), pages 5893-5900. IEEE, 2019.

[22] Ziyan Wang, Buyu Liu, Samuel Schulter, and Manmohan Chandraker. A parametric top-view representation of complex road scenes. In Proceedings of the IEEE Conference on Computer Vision and Pattern Recognition, pages 1032510333, 2019.

[23] Bin Yang, Wenjie Luo, and Raquel Urtasun. PIXOR: Realtime $3 \mathrm{~d}$ object detection from point clouds. In Proceedings of the IEEE conference on Computer Vision and Pattern Recognition, pages 7652-7660, 2018.

[24] Gengshan Yang, Joshua Manela, Michael Happold, and Deva Ramanan. Hierarchical deep stereo matching on highresolution images. In Proceedings of the IEEE Conference on Computer Vision and Pattern Recognition, pages 55155524, 2019.

[25] Yurong You, Yan Wang, Wei-Lun Chao, Divyansh Garg, Geoff Pleiss, Bharath Hariharan, Mark Campbell, and Kilian Q Weinberger. Pseudo-LiDAR++: Accurate depth for 
$3 \mathrm{D}$ object detection in autonomous driving. arXiv preprint arXiv:1906.06310, 2019.

[26] Feihu Zhang, Victor Prisacariu, Ruigang Yang, and Philip HS Torr. GA-Net: Guided aggregation net for endto-end stereo matching. In Proceedings of the IEEE Conference on Computer Vision and Pattern Recognition, pages 185-194, 2019.

[27] Zhi-Hua Zhou. Ensemble methods: foundations and algorithms. CRC press, 2012. 